Planetary Systems in the Universe - Observation, Formation and Evolution

Proceedings IAU Symposium No. 202, (C)2004 IAU

Alan Penny, Pawel Artymowicz, Anne-Marie Lagrange, 8 Sara Russell, eds.

\title{
HD 83443: a system with two Saturns
}

\author{
M. Mayor, D. Naef, F. Pepe, D. Queloz, N.C. Santos, S. Udry and \\ M. Burnet
}

Observatoire de Genève, CH-1290 Sauverny, Switzerland

\begin{abstract}
We report the discovery of an extrasolar planetary system with two Saturnian planets around the star HD 83443. The new planetary system is unusual by more than one aspect, as it contains two very low-mass gaseous giant planets, both on very tight orbits. Among the planets detected so far, the inner planet has the smallest semi-major axis (0.038 AU) and period (2.985 days) whereas the outer planet is the lightest one with $\mathrm{m}_{2} \sin i=0.53 \mathrm{M}_{\text {Sat }}$. A preliminary dynamical study confirms the stability of the system.
\end{abstract}

\section{Introduction}

During the last 5 years, about 50 exoplanets have been detected around stars of the solar vicinity, but only one system with multiple planets has been unambiguously identified around the star $\nu$ And (Butler et al. 1999; Marcy et al., this volume). In addition to a new planet + very low-mass brown dwarf system orbiting HD 168443 (Udry, Mayor \& Queloz, this volume), we report here the discovery of a new extra-solar planetary system with two Saturnian planets around the star HD 83443.

Table 1. Best Keplerian orbital solutions and inferred parameters

\begin{tabular}{lrcc}
\multicolumn{2}{r}{ Parameter } & HD 83443 b & HD 83443 c \\
\hline$P$ & days & $2.9853 \pm 0.0009$ & $29.83 \pm 0.18$ \\
$T$ & HJD-2 400000 & $51386.50 \pm 0.14$ & $51569.59 \pm 0.73$ \\
$e$ & & $0.079 \pm 0.033$ & $0.42 \pm 0.06$ \\
$\gamma$ & $\mathrm{km} \mathrm{s}^{-1}$ & $28.917 \pm 0.001$ & $\ldots$ \\
$\omega$ & $\circ$ & $300.25 \pm 17.05$ & $337.42 \pm 10.42$ \\
$K_{1}$ & $\mathrm{~m} \mathrm{~s}^{-1}$ & $56.1 \pm 1.4$ & $13.8 \pm 1.0$ \\
$f_{1}(m)$ & $10^{-12} \mathrm{M}_{\odot}$ & 54 & 6.05 \\
$m_{2} \sin i$ & $\mathrm{M}_{\mathrm{Jup}}$ & 0.34 & 0.16 \\
$a$ & $\mathrm{AU}$ & 0.038 & 0.17 \\
$N$ & & \multicolumn{3}{c}{93} \\
$\sigma(O-C)$ & $\mathrm{m} \mathrm{s}^{-1}$ & & \\
\hline \hline
\end{tabular}

This new system was detected by means of high-precision radial-velocity measurements with the CORALIE high-resolution spectrograph at the 1.2-meter Leonard Euler telescope (ESO La Silla, Chile) as part of the Geneva planet- 

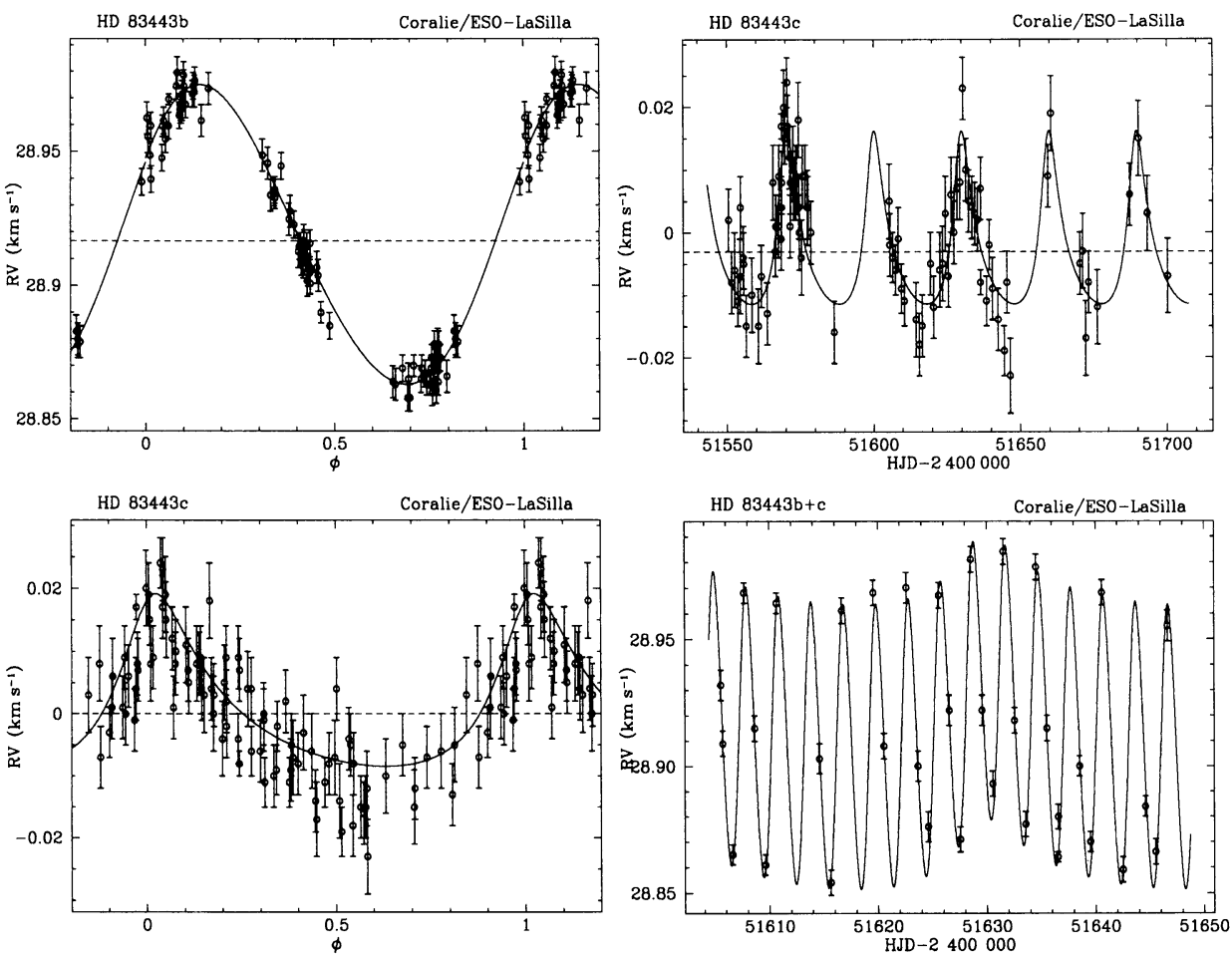

Figure 1. HD 83443. Top left: phased short-period orbit. Top right: residuals of the short-period orbit with the long-period solution. Bottom left: phased long-period orbit. Bottom right: part of the data with the combined solutions

search project (Udry et al. 2000) in the southern hemisphere. HD 83443 is a $\mathrm{K} 0 \mathrm{~V}$ star of $0.79 \mathrm{M}_{\odot}$ at $23 \mathrm{pc}$ from the Sun.

\section{The new system}

The short-period planet was already announced on May 4th, 2000 (ESO Press Release, http://www.eso.org/outreach/press-rel/pr-2000/pr-13-00.html). This inner planet is the planet with the shortest period and with the smallest separation from the parent star among the known candidates. Its period $\mathrm{P}=2.9853$ days is very close to an integer expressed in sideral time: 2.9935 sidereal days. In consequence almost the same radial velocity is observed every 3 days and several months will be needed to fill the different phases of the orbit. After a whole season of measurements we still notice a clumpiness of the observations at three phases (Fig. 1, top left).

Very early an abnormal behaviour had been detected in the residuals of the short-period Keplerian solution showing an apparent periodicity of about one month (Fig. 1, top right). New measurements added after the announcement of HD $83443 \mathrm{~b}$ have fully confirmed the presence of the second planet (Fig. 1, bottom left). HD $83443 \mathrm{c}$ is the planet with the smallest projected mass discov- 


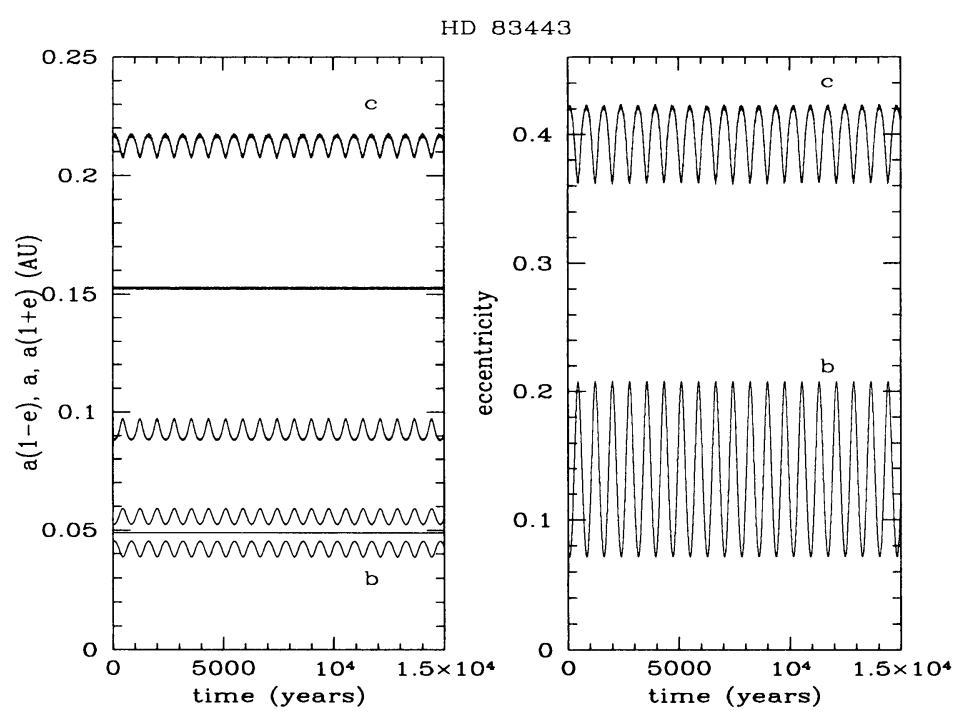

Figure 2. Temporal evolution of the separation and eccentricity parameters for the two planets of the HD 83443 system

ered to date. The system HD 83443 is thus composed of 2 planets with very low masses, respectively close to and smaller than the mass of Saturn.

The two period ratio $\left(r=P_{\mathrm{c}} / P_{\mathrm{b}}=9.9923\right)$ is very close to 10 but the uncertainty on this value is still rather large $(0.063)$. More observations during the coming season are required to check that this ratio is really an integer value.

\section{The dynamical stability of the system}

Preliminary analyses of the dynamical stability have been independently done by J. Laskar and W. Benz (Fig. 2). Integration on a span of about $10^{5}$ years has confirmed the stability of the system despite the strong dynamical interaction between the two planets visible in Fig. 2 as variations of the eccentricity and separation parameters. In particular, the eccentricities of the two orbits are anti-correlated. The significant non-circularity of the inner orbit is explained by the outer body perturbation.

\section{References}

Butler R.P., Marcy G.W., Fischer D.A., et al. 1999, ApJ, 526, 916

Udry, S., Mayor, M., Naef, D., et al. 2000, A\&A, 356, 590 